\title{
The Effects of Gay Sexually Explicit Media on the HIV Risk Behavior of Men who have Sex with Men
}

\author{
B. R. Simon Rosser ${ }^{1}$, Derek J. Smolenski ${ }^{1}$, Darin Erickson ${ }^{1}$, Alex lantaffi ${ }^{1}$, Sonya S. \\ Brady ${ }^{1}$, Dylan L. Galos ${ }^{1}$, Jeremy A. Grey ${ }^{1}$, Gert Martin Hald ${ }^{2}$, Keith J. Horvath ${ }^{3}$, Gunna \\ Kilian ${ }^{1}$, Bente Træen ${ }^{4}$, and J. Michael Wilkerson ${ }^{5}$ \\ ${ }^{1}$ Department of Epidemiology and Community Health, University of Minnesota, Minneapolis, \\ Minnesota \\ ${ }^{2}$ Department of Public Health, University of Copenhagen, Denmark \\ ${ }^{3}$ Department of Psychiatry \& Behavioral Medicine, Medical College of Wisconsin, Milwaukee, \\ Wisconsin \\ ${ }^{4}$ Department of Psychology, University of Tromsø, Denmark \\ ${ }^{5}$ School of Public Health, University of Texas, Houston, Texas
}

\begin{abstract}
This study sought to study consumption patterns of gay-oriented sexually explicit media (SEM) by men who have sex with men (MSM); and to investigate a hypothesized relationship between gay SEM consumption and HIV risk behavior. Participants were 1391 MSM living in the US, recruited online to complete a SEM consumption and sexual risk survey. Almost all (98.5\%) reported some gay SEM exposure over the last 90 days. While $41 \%$ reported a preference to watch actors perform anal sex without condoms (termed "bareback SEM"), 17\% preferred to actors perform anal sex with condoms (termed "safer sex SEM") and 42\% reported no preference. Overall SEM consumption was not associated with HIV risk; however participants who watched more bareback SEM reported significantly greater odds of engaging in risk behavior. The results suggest that a preference for bareback SEM is associated with engaging in risk behavior. More research to understand how MSM develop and maintain preferences in viewing SEM, and to identify new ways to use SEM in HIV prevention, is recommended.
\end{abstract}

\section{Keywords}

gay; pornography; cyberpornography; HIV prevention; Internet; unsafe sex

\section{Introduction}

This study sought, first, to study consumption patterns of gay-oriented sexually explicit media (SEM) by men who have sex with men (MSM); and second, to investigate a hypothesized relationship between gay SEM consumption and HIV risk behavior. While older studies used the term "pornography" for SEM, more recent studies $(1,2)$, including those by our team, have replaced "pornography" with SEM, given that the terms "pornography" or "porn" have become value-laden with highly negative connotations for some people (3). In defining SEM, we use Hald's (4) definition as "any kind of material

Corresponding author: B.R. Simon Rosser, Professor and Director, HIV/STI Intervention \& Prevention Studies (HIPS) Program, Division of Epidemiology \& Community Health, University of Minnesota School of Public Health, 1300 South Second Street, \#300, Minneapolis, MN 55454, 612-624-0358, Fax: 612-624-0315, rosser@umn.edu. 
aiming at creating or enhancing sexual feelings or thoughts in the recipient and, at the same time, containing explicit exposure and/or descriptions of the genitals and clear and explicit sexual acts."

Visual depictions of male-male sex date back at least 2,500 years (5). Commentators note SEM has "always had an exalted position in gay culture" (6), is highly acceptable to gay men (7), and ubiquitous in the gay community (8). Economically, in 2007, it was estimated that gay SEM constituted 33-50\% of all revenue generated by the adult industry $(6,8,9)$. Extrapolating these figures, in the US the gay SEM market is a $\$ 1.3-6.5$ billion industry annually (3). Producers of gay SEM claim its role in validating homosexuality, creating an outlet for desire and exploration, and strengthening community (10). Other researchers have noted that SEM may play a positive role in young MSM's development and sexual education (11-13), with young MSM describing SEM as a major source of sexual information (14).

While these papers have highlighted the benefits of SEM, others have expressed concern at potential negative health effects. In particular, Tydén and Rogala (15) speculate on the effects of gay SEM consumption on HIV risk behavior. "All the [HIV prevention] efforts to modify sexual behavior by increasing condom use and increasing risk awareness may be jeopardized by the global pornography industry through its efficient distribution channels, such as internet, cable television and videos, where amongst others, 'unsafe sex' is promoted.” (p. 590).

Compared to heterosexual SEM, gay SEM is more likely to depict condoms (16). In the late 1980s, the major gay SEM producers in the USA committed to show all anal sex between men depicting condoms $(17,18)$. This self-imposed industry standard lasted for about a decade before the re-emergence of SEM depicting unprotected anal sex between men, dubbed "bareback SEM" (19). Since then, the use or non-use of condoms in gay SEM has remained controversial, with industry safer sex advocates arguing to retain the standard both to protect actors and to model safer sex behavior, while others argue that consumer demand and competition from amateur SEM producers necessitate bareback production (20).

Little empirical research has investigated the effects of gay sexually explicit media (SEM) on the health and HIV risk behavior of men who have sex with men (MSM) (3). We lack solid empirical data that enumerates what effect, if any, gay SEM consumption has on HIV risk behavior as only a handful of studies have been published on the association between SEM and HIV sexual risk behaviors or attitudes in MSM. In these studies SEM consumption or specific SEM genres have been found to be positively associated with finding anal sex activities appealing, having sex with two or more men at the same time and engaging in unprotected anal intercourse $(8,13,21-23)$. Further, use of SEM during partnered sex among MSM has been found to be negatively correlated with condom use during first intercourse with the most recent partner and positively correlated with experience with group sex (24). Major shortcomings pertaining to this previous research include small sample sizes, highly biased sampling procedures, unusual eligibility criteria, restriction of samples to certain local urban areas rather than nationwide, focus on attitudes rather than behavior, measurement of SEM exposure as times (versus duration), a failure to differentiate between accidental and intentional exposure $(3,8,23)$.

In the largest study to date, Stein et al.(23) investigated SEM consumption in "high risk" MSM in New York $(N=2,552)$. After excluding men in monogamous relationships and those not reporting anal intercourse, 821 (32\%) provided both information on their SEM consumption and risk behavior with casual male partners. Almost all participants reported viewing gay SEM (99\%), with 95\% reporting seeing depictions of protected anal intercourse 
(PAI) and 94\% reporting viewing unprotected anal intercourse (UAI). The median time spent viewing gay SEM per week was 60 minutes. The media used for gay SEM were the Internet (96\%), digital video disks or DVDs $(57 \%)$, magazines $(18 \%)$ and other media $(2 \%)$. Over half $(55 \%)$ reported that viewing SEM influenced their sexual behavior, with increasing percentages of viewing UAI in gay SEM associated with increasing odds of engaging in UAI. The authors concluded that viewing SEM depicting UAI was significantly associated with engaging in UAI for high risk MSM. Limitations of the study included partial recruitment from gay SEM sites, the study having unusual eligibility criteria (restricting the sample to high risk MSM), and the study being limited to one urban epicenter, all of which may limit generalizability of findings.

Research on preferences in viewing safer sex versus bareback SEM has the potential to advance our understanding of the reasons why MSM engage in safer sex and unsafe sex. From script theory (25), to the extent that viewing SEM may influence preferences for sex, depictions of safer sex may prompt or reinforce consistent condom use and depictions of unsafe sex may prompt or reinforce inconsistent or non-condom use. The most commonly cited reasons for condom use in actual sexual encounters include prevention of HIV and sexually transmitted diseases $(26,27)$ and for $\operatorname{HIV}$-positive men, altruism $(26,28)$. The most commonly cited reasons for non-condom use include personal pleasure, physical sensation and a belief that condoms spoil sex $(27,29,30)$. But personal protection or pleasure do not explain preferences for viewing or not viewing condoms in gay SEM. Thus, to advance long-term HIV prevention strategies, we were interested in discovering whether MSM have preferences for or against viewing condoms in gay SEM. In addition we were interested in what relationship, if any, exists between such preferences and HIV risk.

Consequently, the aims of this study were two-fold. The first aim was to study exposure to and consumption of gay-oriented SEM by MSM. A priori, we hypothesized that MSM would be high SEM consumers, which we defined as almost all (>95\%) MSM reporting some exposure to gay SEM and most (>80\%) reporting recent consumption (last 90 days). Further, we predicted most MSM would report having watched both SEM depicting safer sex and bareback SEM.

The second aim was to study the relationship between SEM consumption and HIV risk behavior. Here, we predicted that in crude analysis, there would be an overall significant relationship between SEM consumption and HIV risk behavior. It was hypothesized that men engaging in unprotected anal intercourse with multiple male partners (UAIMP) in the last 90 days, would report more SEM consumption in the last 90 days, than men who do not report UAIMP. It was also hypothesized that MSM who reported watching bareback SEM in the last 90 days, would report more UAIMP than men who watched only safer sex SEM. The crude association between SEM and HIV risk behavior was predicted to become nonsignificant after adjusting for type of SEM (bareback vs. safer sex) watched.

\section{Methods}

\section{Study design}

The Sexually Explicit Media (SEM) study was a large, cross-sectional, Internet-based survey of men who have sex with men conducted between May and August, 2011. The survey was designed to collect data on exposure to SEM, sexual behavior, and psychosocial factors associated with HIV transmission risk behavior. Participants were recruited through banner advertisements placed on 148 gay-oriented websites through the Gay Ad Network. A total of 7,939,758 impressions were displayed during this period; banners yielded a clickthrough-rate of $0.16 \%$. An eligibility screener restricted participation to those identifying themselves as male, at least 18 years of age, who reported at least one male sexual partner in 
the last five years, and who were living in the US or one of its territories. Participants were quota-sampled by race/ethnicity to increase diversity in the sample. A total of 5,201 MSM met the eligibility criteria (excluding racial caps). By design, to ensure a racially/ethnically diverse sample, 3,338 MSM were excluded because that racial/ethnic category had filled, leaving a total of 1,863 MSM who met all eligibility criteria. Of these, 1,479 (79.4\%) consented to participate in the study and provided information on exposure to pornography. After excluding 88 participants for impossible or nonsensical data patterns on sexual behavior data, the final sample size for this study was 1,391 . The average completion time for the survey was 42 minutes, and participants were compensated $\$ 25$. All study protocols and consent procedures were approved by the Institutional Review Board at the investigators' home institution.

\section{Measures}

Exposure to SEM-Exposure to SEM was measured in terms of content preference for protected and unprotected anal intercourse, the frequency of exposure to protected and unprotected anal intercourse, and the typical frequency and duration of exposure to SEM of any kind. The preference for viewing condom use during anal intercourse in SEM was measured by a single item, "In general, do you prefer to watch actors perform anal sex with condoms or without?" with three nominal response options: (1) without condoms, (2) with condoms, and (3) I do not care either way. Participants also responded to two 5-point, Likert-type items on the frequency of viewing protected and unprotected anal intercourse when they watched SEM during the past 3 months. The response range was from $1=$ "rarely or never" to $5=$ "always or almost always." We created an index by subtracting the frequency of viewing protected anal intercourse from the frequency of viewing unprotected anal intercourse to provide an ordinal measure of the tendency to view unprotected anal intercourse versus protected anal intercourse. This index ranged from -4 to 4 , with -4 indicating exclusive viewing of protected anal intercourse and 4 indicating exclusive viewing of unprotected anal intercourse. A score of zero indicated equivalent exposure to both forms of anal intercourse. Finally, frequency and duration measures of SEM consumption of any kind in the last three months were combined to create an index of the hours per week dedicated to SEM consumption.

In addition, four items were used to assess the frequency of accessing SEM through the following four sources: (1) magazines, (2) video/DVD, (3) Internet on a computer, and (4) Internet through a phone or mobile device. Response options to each of these items ranged from $1=$ not at all to $6=$ more than once a day. One item asked participants to report the typical duration of use of SEM when it was used in the last 90 days, with response categories including: (1) 1 - 15 minutes, (2) 16 - 30 minutes, (3) $30-45$ minutes, (4) $46-$ 60 minutes, (5) between 1 and $1 \frac{1 / 2}{2}$ hours, (6) between $1 \frac{1 / 2}{2}$ and 2 hours, and (7) more than 2 hours.

Positive and Negative Affect Schedule (PANAS)—The PANAS (31) was used to assess positive and negative affect in the last 90 days. The measure involves 10 adjectives. For each adjective, participants were asked to indicate the extent to which they felt that way during the past 90 days. All items were responded to using a 5-point Likert-type index, with $1=$ "very little or not at all", and $5=$ "extremely." We used arithmetic means of the five positive (e.g., "excited") and five negative (e.g., "scared") items to create two composite measures of positive and negative affect. In this sample, the Cronbach alpha is 0.82 for positive affect and the Cronbach alpha is 0.87 for negative affect.

Social Desirability-We used the Marlowe-Crowne short-form (32) to measure social desirability. The measure included 10 true/false statements about general characteristics of 
the participants. Responses were coded 1 for providing the desirable answer to each item.

We created a composite measure by summing the number of desirable responses, resulting in a summary measure ranging from 0 to 10 in this sample. Sample items include, "I can remember playing sick to get out of something" (reverse-coded) and "I am always courteous, even to people who are disagreeable." The Kuder-Richardson 20 internal consistency estimate for this measure was 0.59 .

Compulsive Sexual Behavior Inventory (CSBI)—We used the "control" subscale of the CSBI to assess compulsive, or out-of-control sexual behavior (33). The subscale comprised 13 items measured using 5-point Likert-type response scales with $1=$ "Very frequently" and 5 = "Never." Sample items include, "How often have you had trouble controlling your sexual urges" and "How often have you used sex to deal with problems or worries in your life?" To create a composite, we calculated the arithmetic mean across the 13 items and reversed the valence of this average so that higher scores on the composite measure indicated more frequent out-of-control behavior. Cronbach's alpha in this sample was 0.90 .

Internalized Homonegativity (IH)—We used the revised Reactions to Homosexuality scale (34) to measure internalized homonegativity. The measure comprised seven, 7-point, Likert-type items to assess the degree to which the items corresponded with the respondents' perceptions of themselves. Sample items include, "I feel comfortable discussing homosexuality in a public situation" and "Even if I could change my sexual orientation, I wouldn't." The response options ranged from 1 = "Strongly disagree" to 7 = "Strongly agree." We used the arithmetic mean to develop a composite for this measure that was valenced so that higher scores indicated more internalized homonegativity. Cronbach's alpha in this sample was 0.83 .

Sexual Behavior-Participants were asked to report the number of casual unprotected anal intercourse male partners (UAIMP) in the last 90 days. We asked participants to provide this count separately for partners with whom they engaged in insertive (UAIMP-I) and receptive (UAIMP-R) anal intercourse. In addition, participants reported the number of UAIMP that were HIV-negative, HIV-positive, or unknown. Using each participant's selfreported HIV-status, we developed a binary indicator of whether or not the participant had engaged in serodiscordant or potentially serodiscordant unprotected anal intercourse. HIVnegative participants who reported any HIV-positive or unknown UAIMP, and HIV-positive participants who reported any HIV-negative or unknown UAIMP were classified as engaging in serodiscordant or potentially serodiscordant unprotected anal intercourse (SDUAI).

Demographic Characteristics-Demographic questions were asked wherever possible using wording from the Census (2010). Age was measured continuously. Race/ethnicity was measured using two separate questions, and collapsed at analysis. Education was measured categorically.

Other Characteristics-HIV status was asked with five response options: HIV-positive, HIV-negative, I'm not sure but I think HIV-positive, I'm not sure but I think HIV-negative and Don't know. Being in a long-term relationship was defined as having a "regular sex partner such as a boyfriend, husband, domestic partner that you have been in a relationship with for at least three months," and assessed by asking "How many of the male partners [just identified in the prior question] were primary partners?" It was further cross-validated by asking the length of time in the relationship. Lifetime number of male partners was asked as an open numeric variable, worded as "About how many male sexual partners have you had 
in your lifetime?" Drug use was asked, "In the past 3 months, how often have you used any of the following drugs illegally or inappropriately (e.g., abuse of prescription drugs)?" with response options being not at all, less than monthly, once a month, one a week, daily or refuse to answer. Categories of drugs investigated included, marijuana/hasish, cocaine, uppers (methamphetaminers, crystal), downers (valium, sedatives), club drugs (GHB, ecstasy), opioids (heroin, Vicodin), erectile enhancement drugs (e.g., Viagra, Cialis), and poppers.

\section{Statistical methods}

We used Mplus, Version 6.1 (35) to estimate full information maximum likelihood regression models to address the main hypotheses in this study. In the first set of models, we used all 1,391 participants (including 19 participants who reported never being exposed to SEM) to examine the association between exposure to SEM and the three outcome measures. For the two count outcomes (UAIMP-I and UAIMP-R), we specified a negative binomial link function to account for overdispersion of the variance. For the binary outcome, SDUAI, we specified a logit link function. Exponentiation of resulting coefficients from these models yielded prevalence rate ratios (PRR) and a prevalence odds ratio (POR), respectively. All three outcomes were included simultaneously in the models. All demographic variables included in Table I and the psychosocial measures were included in the model as exogenous covariates based on a priori considerations of their potential to be confounds and to not be on the causal pathway between exposure to SEM and the specified outcomes. We used two formulations of the measure of SEM dose: natural logarithm transformation to examine both a linear and quadratic association, and a four-level categorical variable using cutpoints in the frequency of SEM exposure to facilitate interpretation. For the latter, we specified the categorical measure as nominal to compare different exposure levels to a common referent category, and we ran a separate model in which we specified the measure as ordinal to test for linear trend. We report both the Akaike Information Criterion (AIC) and the Sample-size-adjusted Bayes Information Criterion (SBIC) for each model to compare the relative fit to the data.

We estimated a second set of models after restricting the sample to the 1,372 participants who reported any exposure to SEM to test for effect measure modification of the association between exposure to SEM and the relative amount to which the SEM consumed depicted unprotected anal intercourse. For these models, we used the natural logarithm transformed measure of exposure and the ordinal measure of unprotected versus protected anal intercourse content as main effects and a multiplicative term of the two variables to test for effect measure modification. We examined the statistical significance of the multiplicative terms and the AIC and SBIC of the models with and without the interaction terms to determine the presence or absence of effect measure modification on a multiplicative scale. In these models, we also included the nominal measure of content preference to provide a robust assessment of exposure to SEM (typical exposure consumption, actual viewing of unprotected or protected anal intercourse, and preference for unprotected or protected anal intercourse).

\section{Results}

Demographic characteristics of the participants are displayed in Table I. Similar to previous work with Internet-based samples of MSM, participants were younger, well-educated, gayidentified and mostly HIV negative (36). Unlike other studies, only a minority of participants were non-Hispanic White. More participants were non-white (58.6\%) than is usually observed in Internet-based convenience samples (37). This reflects the quotasampling on race/ethnicity to over-recruit men of color into the study. 
Table II displays the descriptive statistics for the exposures, outcomes, and psychosocial covariates used in the analysis. On average, participants had higher scores on positive affect as compared to negative affect. The measure of social desirability was approximately normally distributed, indicating that social desirability still occurs even in anonymous, Internet-based data collection. The measures of compulsive sexual behavior and internalized homonegativity were positively skewed indicating the majority of participants had low scores on these variables.

In all, $98.5 \%$ participants reported exposure to SEM, during the last 90 days. Confirming the dominance of Internet-mediated SEM, most participants (97.8\%) reported accessing SEM on a computer, followed by video/DVD (45.4\%), then by Internet through a phone or mobile device (42.0\%), and last of all, magazines (19.2\%). The continuous measure of SEM dose in hours per week was positively skewed as evidenced by the median being lower than the mean. Based on the median, the typical dose was almost three hours per week, or 24.9 minutes per day. This is reflected in the categorical classification of dose with the modal category being between one hour and three-and-one-half hours per week. In terms of content viewed, the typical respondent reported equivalent amounts of protected and unprotected anal intercourse as evidenced by the mean being approximately zero and the median being zero. The small negative value of the mean suggests that more participants reported a greater amount of exposure to protected anal intercourse as compared to unprotected anal intercourse. Regarding preferences, $40.2 \%$ reported a preference for bareback SEM, 17.2\% a preference for safer sex SEM, and $42.6 \%$ no preference. This sample reported a low frequency of UAIMP, either insertive or receptive; approximately $10 \%$ of participants reported any serodiscordant UAIMP.

The regression models that examined the association between exposure to SEM and highrisk sexual behavior in all participants (Table III) indicated that a linear model had better fit to the data than a quadratic model (Models 1 and 2). For UAIMP-R, we observed that the quadratic term was statistically-significant; however, the fit indices indicated a preference for the simpler linear model. A higher average dose in terms of hours per week was marginally associated with an increased prevalence rate of UAIMP-I and UAIMP-R. The association between exposure to SEM and a report of SDUAI was stronger. Upon specification of exposure to SEM as a nominal variable, we observed that participants who reported more than seven hours per week of SEM exposure had the strongest associations with UAIMP-R and SDUAI; there was also evidence of a linear trend across the exposure categories associated with these two outcomes, suggesting an increase in the prevalence rate or odds of risk behavior as a function of increasing dose to SEM. Neither the linear trend nor the dose categories were associated with UAIMP-I.

In the final models (Table IV), we did not observe effect measure modification on the multiplicative scale between the continuous measure of exposure to SEM and viewing more unprotected anal intercourse. The associations between the continuous dose measure and risk behavior in Table III were attenuated upon the inclusion of viewing more unprotected anal sex content and the preference for no condom or condoms being used (Model 2). Only UAIMP-R retained a marginal association with overall SEM exposure. Participants who reported viewing more unprotected anal intercourse in SEM as compared to protected anal intercourse had a greater prevalence rate and odds of reporting sexual risk behavior, independent of overall SEM exposure. The strongest associations were observed between the preference for condom use in SEM and risk behavior, with participants who preferred no condoms in anal sex depictions reporting more risk behavior compared to participants with no preference. Those who preferred condoms used in anal sex depictions were at a markedly decreased risk. Specification of the exposure to SEM variable as nominal in these models indicated that no discrete category of exposure was statistically-significant in the association 
with risk behavior; however, for both UAIMP-R and SDUAI, there was a statisticallysignificant linear trend, suggesting an increase in the prevalence rate and odds as a function of increasing levels of exposure.

\section{Discussion}

There are four major limitations to note for this study. First, the cross sectional survey nature of this study prevents exploration of temporality or causality. Second, we lack an adequate literature in which to contextualize the findings or to discuss reliability of results. Third, this survey-based study relies on self-reported data and, in some situations, subjective assessments (e.g., whether SEM depicts bareback or safer sex, and seroconcordance in sex with other partners). Fourth, the study sample was restricted, by design, to Internet-using MSM recruited as a convenience sample. Since most SEM is online, MSM who do not use the Internet may have very different SEM use patterns. Since this is a convenience sample, the generalizability of findings to other Internet-using MSM, or all MSM, is unknown.

To highlight the key findings of this study, recent gay SEM exposure appeared normative for this sample, with most MSM reporting over three hours of SEM exposure per week. Reflecting the dominance of cyber-SEM, almost all participants reported accessing gay SEM via the Internet. Recent HIV risk behavior, whether measured behaviorally (UAIMP) or by taking into account serostatus, was relatively infrequent.

For most MSM, we found no evidence of an association between overall SEM use and HIV risk. However a marginal association between SEM use and HIV risk behavior was observed for those engaging in the highest levels of SEM viewing ( $>1$ hour per day). This indicates a moderating effect of viewing time of SEM on the relationship between SEM and HIV risk behavior. Notably, this association attenuates when other variables are controlled for, highlighting the influence of other factors on the SEM-HIV risk behavior relationship. Most MSM report a preference for or against seeing condoms in SEM. We found a strong association between bareback and safer sex preferences in gay SEM and HIV risk behavior. Compared to MSM with no preferences, those with a preference for watching bareback SEM reported significantly higher risk behavior, while those with a preference for watching safer sex SEM reported significantly lower risk behavior. This finding warrants further investigation.

Why do some men develop preferences for depictions of anal sex with condoms while others have preferences for depictions without? As summarized in the introduction, the existing HIV prevention literature -- which emphasizes self-interest for using protection and pleasure for engaging in bareback sex - cannot adequately explain preferences for watching safer sex or bareback SEM. There are at least three potential explanations to consider. First, consistent with script theory $(38,39)$, perhaps over time, anal sex with condoms becomes eroticized for some men, bareback sex becomes eroticized for others, while still others find other features of SEM more salient. If so, then watching safer sex or bareback SEM may act to reinforce the preferred behavior, for example, through operant conditioning. This explanation is consistent with our formative research findings. Prior to the survey, we conducted focus groups of 79 gay SEM consumers divided into whether they perceived their SEM-consumption as problematic or non-problematic, and whether they preferred viewing safer sex SEM, bareback SEM or had no preference (a $2 \times 3$ focus group design detailed elsewhere $(40,41)$. Overall, we found strong similarities between the self-identified problematic and non-problematic groups, leading us to collapse their data. In the barebackSEM-as-problematic groups, some participants did report that watching bareback SEM made them want to engage in UAI. Hence, for them, bareback SEM could be considered a risk stimulus. Participants in the bareback-preferred-but-non-problematic groups reported 
using bareback SEM as a substitution for engaging in risk, making it a protective factor. These men described liking bareback SEM because they could enjoy sex with no restrictions while not placing themselves at risk. HIV positive participants noted that bareback SEM allowed them to enjoy their fantasies without being reminded of HIV. Still others reported whether the SEM depicted bareback or safer sex was irrelevant to them as they focused on other aspects of the SEM (e.g., actors' looks or the sexual acts themselves).

A second explanation for our main finding is that preferences for or against condoms in SEM is not causally related to engaging in risk behavior, but reflective of some other preferences in SEM or sexual behavior. For example, SEM genre (depictions of regular/ vanilla SEM versus heavier/kink SEM) may influence both whether safer sex or bareback scenes are depicted, and may appeal to different SEM consumers. Hence, a preference for SEM depicting leather sex, group sex, or kink may result in a higher probability of viewing bareback SEM than SEM depicting scenes of mutual masturbation and oral sex. Future studies should control for genre. Finally, a third explanation is that other variable(s) might explain the seemingly contradictory finding that while watching UAI in SEM was weakly associated with increased risk behavior, most MSM reported viewing some UAI in gay SEM while only a small minority reported engaging in actual risk. Are MSM's preferences for bareback or safer sex SEM driving HIV risk behavior, reflecting current practices, or both being driven by other variables? As many individual, social, and SEM variables could potentially mediate the relationship between viewing SEM and actual behavior, more research is needed. Whichever explanation or combination of explanations is true, what emerges from the data is that a simplistic explanation is unlikely to encompass the diversity of MSM's responses to SEM reflected in these results.

Comparing our results with Stein et al.'s study (23), there are five main findings. First, both studies report gay SEM consumption and bareback SEM consumption to be near universal experiences of Internet-using MSM, with both reporting cyber-SEM (by computer or mobile device) as the main way MSM access gay SEM. Researchers in gay men's health need to acknowledge and study SEM consumption as part of the broader context of MSM's sexual lives. Second, neither study found that (overall) SEM consumption is related to HIV risk. Concern that increased gay SEM consumption is fueling the resurgent HIV epidemic among MSM -- as expressed by Tydén and Rogala (15) above -- lacks empirical support. Third, both studies found a relationship between viewing UAI in SEM and engaging in HIV risk behavior, with our study also finding the converse to be true: that viewing protected anal sex is associated with decreased HIV risk behavior. Hence, there is empirical support that bareback SEM consumption is related to risk behavior. Fourth, both studies denote MSM as high SEM consumers. This supports other research which suggests that SEM has an important role in shaping gay sexual norms. Fifth, while Stein et al.'s study (23) of high risk MSM in New York estimated a median time of 60 minutes viewed per week, our sample of MSM recruited nationally reported watching almost three times as much. This suggests that there may be considerable variability in SEM consumption. To reconcile the difference, perhaps high risk MSM in epicenters spend more time pursuing sex, while lower risk MSM recruited more broadly may consume SEM more.

We concur with Stein et al.'s caution (23) against regulation to control UAI in SEM (at least until this relationship is better understood). To the gay SEM industry, we highlight two findings. First, MSM appear to have clear preferences for and against viewing condoms in SEM, confirming the impression that there is consumer-driven demand for both bareback and safer sex SEM. Second, a preference for bareback SEM is associated with increased HIV risk; while a preference for safer sex SEM is associated with lower HIV risk. If these relationships are causal, then the erosion of the "all anal sex with condoms" standard for gay 
SEM production may be having a negative impact on community health by increasing UAI, and ultimately HIV/STI transmission, among MSM.

\section{Conclusions}

We highlight four promising areas of future research. First, researchers interested in studying MSM SEM consumption should include measures of overall SEM consumption, relative measures of SEM depicting unprotected and protected anal sex consumption, and preferences for safer sex or bareback SEM. Second, future research should examine the experience of SEM use in very high consumers (defined as greater than 7 hours per week). While most MSM consume SEM without problems - including without HIV risk -- for a small subgroup of high consumers, our formative research and these results both suggest their behavior may be experienced as problematic. Clinically, it would be helpful to establish what relationship, if any, exists between very high SEM consumption and compulsive sexual behavior, to identify whether the SEM use is compulsive in itself, and then to compare the behavior and characteristics of MSM with and without compulsive SEM use. Third, more research is needed to understand how MSM develop preferences for safer sex SEM or bareback SEM. Since such preferences correlate with HIV risk behavior, a better understanding of the genesis, strength and meaning of such preferences may open up new understandings and interventions for HIV prevention. Finally, with almost universal consumption of gay-SEM by MSM, research should focus on how to use gay SEM for HIV prevention. The structural challenge for HIV prevention is to identify and test ways to use SEM for HIV prevention that are acceptable, feasible and effective.

\section{Acknowledgments}

Understanding Effects of Web-based Media on Virtual Populations was funded by the National Institutes of Mental Health Center for Medical Health Research on AIDS, Grant number 5R01MH087231. All research was carried out with the approval of the University of Minnesota Institutional Review Board, study number 0906S68801.

\section{References}

1. Watney, S. Policing Desire - Pornography, AIDS and the Media. 3. Minneapolis, MN: University of Minnesota Press; 1996.

2. Linz D. Exposure to sexually explicit materials and attitudes towards rape. J Sex Res. 1989; 26:5084.

3. Rosser BRS, Grey JA, Wilkerson JM, et al. A commentary on the role of sexually explicit media (SEM) in the transmission and prevention of HIV among men who have sex with men (MSM). AIDS Behav. 2012; 16(6):1375-81.

4. Hald GM. Gender differences in pornography consumption among young heterosexual Danish adults. Arch Sex Behav. 2006; 35:577-85. [PubMed: 17039402]

5. Souli, S. Love life of the ancient Greeks. Athens, Greece: Toubi’s; 1997.

6. Thomas, JA. Gay male video pornography: Past, present and future. In: Weitzer, R., editor. Sex for sale: Prostitution, Pornography, and the Sex Industry. New York, NY: Routledge; 2000. p. 49-66.

7. Hooper S, Rosser BRS, Horvath KJ, Oakes JM, Danilenko G. Men's INTernet Sex II (MINTS-II) Team. An online needs assessment of a virtual community: What men who use the Internet to seek sex with men want in Internet-based HIV prevention. AIDS Behav. 2008; 12:867-75. [PubMed: 18401701]

8. Morrison TG, Morrison MA, Bradley BA. Correlates of gay men's self-reported exposure to pornography. Intl J Sex Hlth. 2007; 19(2):33-43.

9. Rich, F. Naked capitalists. New York: Times Magazine; 2001 May 20.

10. Lucas M. On gay porn. Yale JL \& Feminism. 2006; 18:299-302. 
11. Kubicek K, Carpineto J, McDavitt B, Weiss G, Kipke M. Use and perceptions of the internet for sexual information and partners: A study of young men who have sex with men. Arch Sex Behav. 2010; 40(4):803-16. [PubMed: 20809373]

12. Kubicek K, Beyer WJ, Weiss G, Iverson E, Kipke MD. In the dark: Young men's stories of sexual initiation in the absence of relevant sexual health information. Health Educ Behav. 2010; 37(2): 243-63. [PubMed: 19574587]

13. Morrison TG. "He was treating me like trash, and I was loving it...": Perspectives in gay male pornography. J Homosex. 2004; 47(3-4):167-83. [PubMed: 15451709]

14. Mustanski B, Lyons T, Garcia SC. Internet use and sexual health of young men who have sex with men: A mixed-methods study. Arch Sex Behav. 2011; 40:289-300. [PubMed: 20182787]

15. Tyden T, Rogala C. Sexual behaviour among men in Sweden and the impact of pornography. Int J STD AIDS. 2004; 15:590-3. [PubMed: 15339365]

16. Grudzen CR, Elliot MN, Kerndt PR, Shuster MA, Brook RH, Gelberg L. Condom use and highrisk sexual acts in adult films: A comparison of heterosexual and homosexual films. Am J Public Health. 2009; 99(S1):S152-S6. [PubMed: 19218178]

17. Calvert C, Richards RD. Gay pornography and the first amendment: Unique, first-person perspectives on free experession, sexual censorship, and cultural images. Am U J Gender Soc Pol'y \& L. 2007; 15(4):688-731.

18. Clark-Flory T. Must porn stars get tested? Salon. 2012 Sep 8.

19. Holt, M. HIV scandal in the gay porn industry. BBC Two: Newsnight [Internet]. 2008 Nov 30. 2012 Available from: http://news.bbc.co.uk/2/hi/programmes/newsnight/7277000.stm

20. McNeil, JDG. Unlikely model in HIV efforts: Sex film industry. New York Times [Internet]. 2012 Nov 30. Available from: http://www.nytimes.com/2012/11/06/health/unlikely-model-for-hivprevention-porn-industry.html?pagewanted=all

21. Duggan S, McCreary DR. Body image, eating disorders, and the drive for muscularity in gay and heterosexual men: The influence of media images. J Homosex. 2004; 47(3/4):45-58. [PubMed: 15451703]

22. Parsons JT, Kelly BC, Bimbi DS, Muench F, Morgenstern J. Accounting for the social triggers of sexual compulsivity. J Addict Dis. 2007; 26:5-16. [PubMed: 18018804]

23. Stein D, Silvera R, Hagerty R, Marmor M. Viewing pornography depicting unprotected anal intercourse: Are there implications for HIV prevention among men who have sex with men. Arch Sex Behav. 2012; 41(1):411-9. [PubMed: 21755381]

24. Træen B, Daneback K. The use of pornography and sexual behavior among Norwegian men and women of differing sexual orientation. Sexologie [Internet]. 2012

25. Simon W, Gagnon JH. Sexual scripts: permanence and change. Arch Sex Behav. 1986; 15(2):97120. [PubMed: 3718206]

26. Harawa NT, Williams JK, Ramamurthi HC, Bingham TA. Perceptions towards condom use, sexual activity, and HIV disclosure among HIV-positive African American men who have sex with men: Implications for heterosexual transmission. J Urban Health. 2006; 83(4):682-94. [PubMed: 16736115]

27. Caballo-Dieguez A, Bauermeister J. "Barebacking": Intentional condomless anal sex in HIV-risk contexts. Reasons for and Against It. J Homosex. 2004; 47(1):1-16.

28. Brennan DJ, Welles SL, Ross MW, Miner MH, Mayer KH, Rosser BRS. Development of a treatment optimism scale for HIV-positive gay and bisexual men. AIDS Care. 2009; 21(9):42-9. [PubMed: 19085219]

29. Offir JT, Fisher JD, Williams SS, Fisher WA. Reasons for inconsistent AIDS-preventive behaviors among gay men. J Sex Res. 1993; 30(1):62-9.

30. Valdiserri RO, Lyter D, Leviton LC, Callahan CM, Kingsley LA, Rinaldo CR. Variables influencing condom use in a cohort of gay and bisexual men. Am J Public Health. 1988; 78:801-5. [PubMed: 3260081]

31. Thompson ER. Development and validation of an internationally reliable short-form of the Positive and Negative Affect Schedule (PANAS). J Cross Cult Psychol. 2007; 38(2):227-42.

32. Strahan R, Gerbasi KC. Short, homogeneous versions of the Marlow-Crowne social desirability scale. J Clin Psychol. 1972; 28:191-3. 
33. Coleman E, Miner M, Ohlerking F, Raymond N. Compulsive sexual behavior inventory: A preliminary study of reliability and validity. J Sex Marital Ther. 2001; 27(2):325-32. [PubMed: 11441516]

34. Smolenski DJ, Diamond P, Ross MW, Rosser BRS. Revision, criterion validity, and multi-group assessment of the Reactions to Homosexuality Scale. J Pers Assess. 2010; 92(6):568-76. [PubMed: 20954058]

35. Muthén, B.; Muthén, LM. plus (Version 6.1). Los Angeles: Muthén \& Muthén; 2009.

36. Rosser BRS, Oakes JM, Horvath KJ, Konstan JA, Danilenko GP, Peterson JL. HIV sexual risk behavior by men who use the Internet to seek sex with men: Results of the Men's INTernet Sex Study-II (MINTS-II). AIDS Behav. 2009; 13(3):488-98. [PubMed: 19205866]

37. Rosser BRS, Wilkerson JM, Smolenski D, et al. The future of Internet-based HIV prevention: A report on key findings from the Men's INTernet Sex (MINTS-I, II) Studies. AIDS Behav. 2011; 15(1):91-100.

38. Gagnon JH. The explicit and implicit use of the scripting perspective in sex research. Annu Rev Sex Res. 1990; 1:1-43.

39. Gagnon, JH.; Simon, W. Sexual Conduct. Chicago, IL: Aldine; 1973.

40. Wilkerson JM, Iantaffi A, Smolenski DJ, et al. The SEM risk behavior (SRB) model: A new conceptual model of how pornography influences the sexual intentions and HIV risk behavior of MSM. Sex Relation Ther. 2012; 27(3):217-30. [PubMed: 23185126]

41. Iantaffi, A.; Wilkerson, JM.; Grey, JA.; Rosser, BRS. The acceptability of sexually explicit messages in HIV prevention among men who have sex with men. Submitted for publication 


\section{Table I}

Characteristics of the sample ( $N=1,391$ Internet-using MSM)

\begin{tabular}{|c|c|c|c|}
\hline Variable & $\mathbf{n}$ & $\%$ & \\
\hline \multicolumn{4}{|l|}{ Age } \\
\hline $18-24$ & 521 & 37.46 & \\
\hline $25-34$ & 440 & 31.63 & \\
\hline $35-44$ & 203 & 14.59 & \\
\hline$\geq 45$ & 227 & 16.32 & \\
\hline \multicolumn{4}{|l|}{ Race/ethnicity } \\
\hline White $^{1}$ & 576 & 41.41 & \\
\hline Black $^{1}$ & 161 & 11.57 & \\
\hline Latino & 421 & 30.27 & \\
\hline Asian/Pacific Islander 1 & 104 & 7.48 & \\
\hline Native American 1 & 24 & 1.73 & \\
\hline Other $1 /$ Multi & 105 & 7.55 & \\
\hline \multicolumn{4}{|l|}{ Education } \\
\hline Up to high school & 157 & 11.29 & \\
\hline Some college & 561 & 40.33 & \\
\hline College graduate & 397 & 28.54 & \\
\hline Postgraduate & 275 & 19.77 & \\
\hline Missing & 1 & 0.07 & \\
\hline \multicolumn{4}{|l|}{ HIV status ${ }^{\dagger}$} \\
\hline Positive & 121 & 8.70 & \\
\hline Negative & 1,269 & 91.23 & \\
\hline Missing & 1 & 0.07 & \\
\hline \multicolumn{4}{|l|}{ Long-term relationship } \\
\hline No & 620 & 44.57 & \\
\hline Yes & 397 & 28.54 & \\
\hline Missing & 374 & 26.89 & \\
\hline \multicolumn{4}{|l|}{ Drug use, last 90 days } \\
\hline No & 751 & 53.99 & \\
\hline Yes & 430 & 30.91 & \\
\hline Missing & 210 & 15.10 & \\
\hline \multicolumn{4}{|c|}{ Lifetime number of male sexual partners } \\
\hline$<20$ & 602 & 43.28 & \\
\hline$\geq 20$ & 616 & 44.28 & \\
\hline Missing & 173 & 12.44 & \\
\hline \multicolumn{4}{|c|}{ Note: $\mathrm{SEM}=$ sexually-explicit media, UAIMP = unprotected male anal intercourse partners } \\
\hline Non-Hispanic & & & \\
\hline
\end{tabular}


${ }^{\dagger}$ For this analysis, HIV status was collapsed to compare those who have tested HIV positive by self-report with all others, including HIV negative and unsure. 


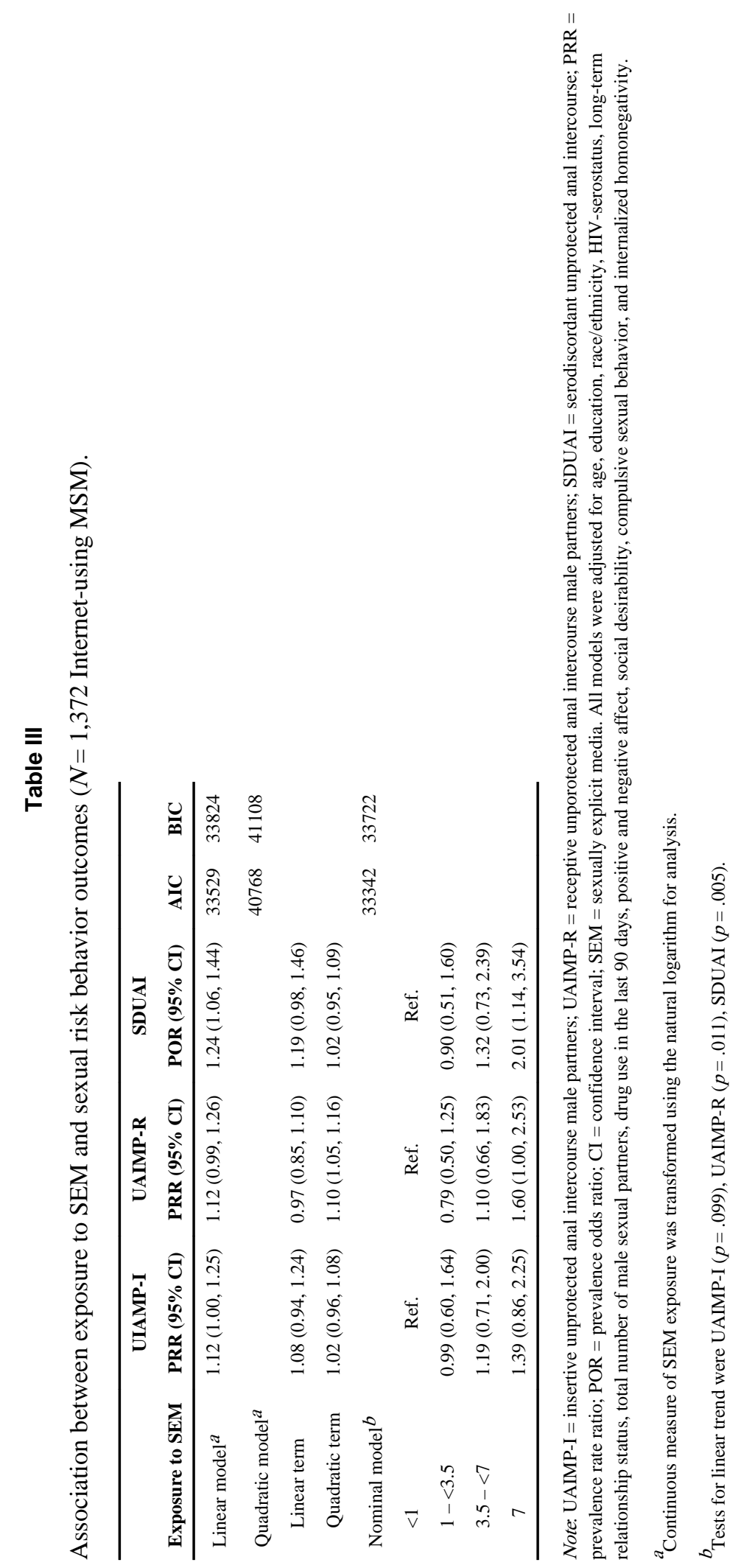

AIDS Behav. Author manuscript; available in PMC 2014 May 01. 


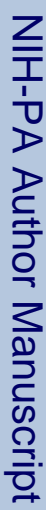

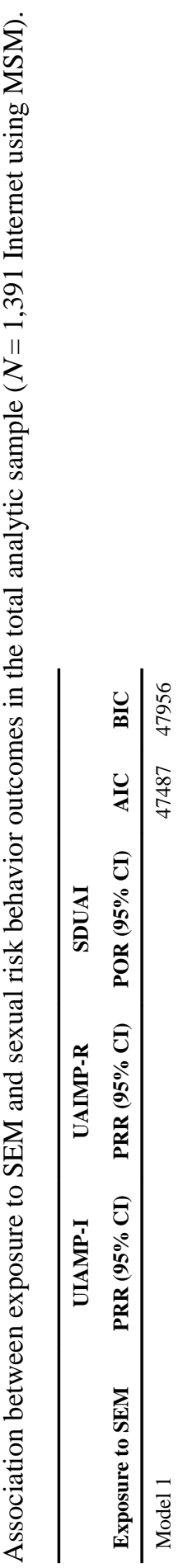

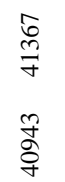

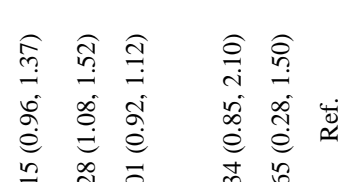

$\stackrel{\infty}{=} \stackrel{\square}{\square}$

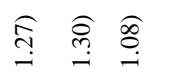

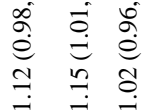

$\stackrel{9}{g} \stackrel{0}{=}$

स ज त व

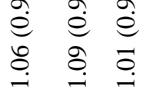

त̂

तू है

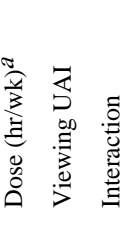

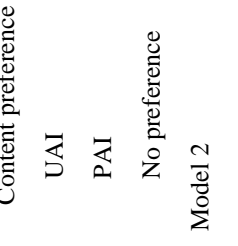

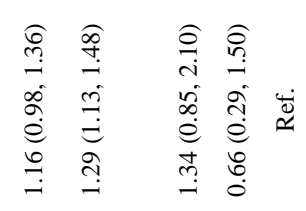

$\frac{\bar{f}}{\text { 子 }}$

ஜ̊̊̆

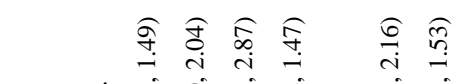

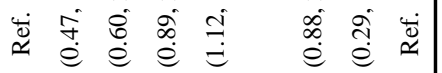

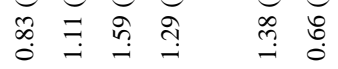

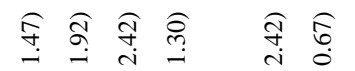

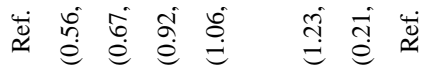

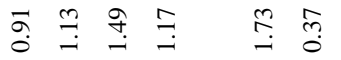

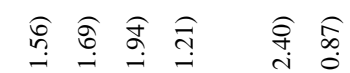

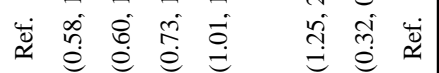

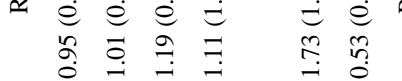




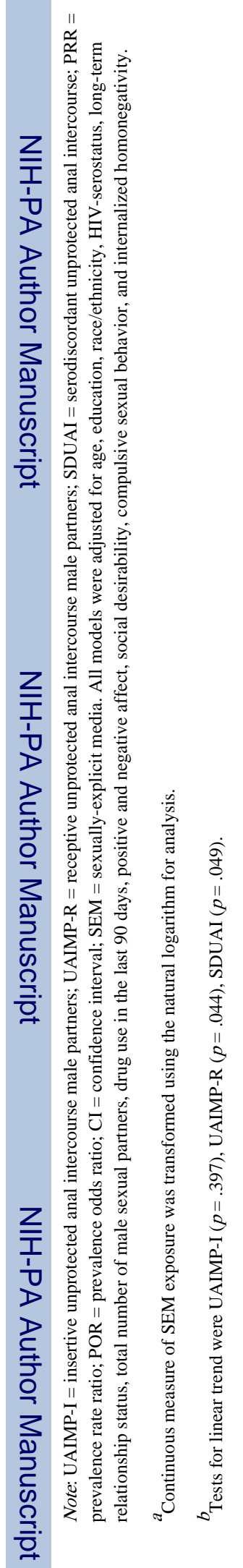

AIDS Behav. Author manuscript; available in PMC 2014 May 01. 